$$
\begin{aligned}
& \text { CONF-9611113--3 } \\
& \text { ANL/ET/CP--915/8 }
\end{aligned}
$$

Measurement of Shear Impedances of Viscoelastic Fluids*

b y

Shuh-Haw Sheen, Hual-Te Chien, and A. C. Raptis

Energy Technology Division

Argonne National Laboratory

Argonne, Illinois 60439

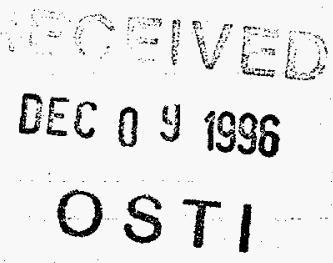

The submitted manuscript has been authored by a contractor of the U.S. Government under contract No. W-31-109-ENG-38. Accordingly, the U.S. Government retains a nonexclusive, royalty-free license to publish or reproduce the published form of this contribution, or allow others to do so, for U.S. Government purposes.

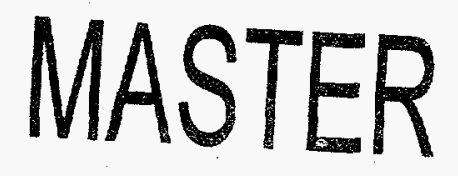

For presentation at 1996 IEEE International Ultrasonics Symposium, November 3-6, San Antonio, TX.

*Work supported by the Office of Environmental Management, U.S. Department of Energy, under Contract W-31-109-ENG-38 


\section{DISCLAIMER}

Portions of this document may be illegible in electronic image products. Images are produced from the best available original document. 


\title{
Measurement of Shear Impedances of Viscoelastic Fluids*
}

\author{
$S h^{1} u h-H a w$ Sheen, Hual-Te Chien, and A. C. Raptis \\ Energy Technology Division \\ Argonne National Laboratory
}

\begin{abstract}
Shear-wave reflection coefficients from a solid/fluid interface are derived for nonNewtonian fluids that can be described by Maxwell, Voigt, and power-law fluid models. Based on model calculations, we have identified the measurable effects on the reflection coefficients due to fluid non-Newtonian behavior. The models are used to interpret the viscosity data obtained by a technique based on shear impedance measurement.
\end{abstract}

\section{Introduction}

Shear impedance of a fluid can be determined by measuring the complex shear-wave reflection coefficient at the solid/fluid interface. ${ }^{1-3}$ Fluid shear viscosity can then be deduced from the shear impedance if shear rate and fluid density can be measured. It has been shown that fluid density can be determined from the acoustic impedance measurement, which uses longitudinal waves. ${ }^{4}$ Recently, we developed a nonintrusive ultrasonic viscometer that simultaneously measures both longitudinal-and shear-wave reflection. coefficients. ${ }^{5}$ Normal-incidence solid wedges are used in our design. The viscometer can also measure sound velocity in the fluid, from which fluid density is determined independently from viscosity measurement. Because the shear-wave reflection coefficient is related to shear impedances of both the fluid and the wedge, the sensitivity and measurement range of this technique as applied to viscosity measurement depend on the wedge property. A wedge of greater shear impedance covers a wider measurement range but with less sensitivity. It is also noticed that reflection coefficients of Newtonian fluids are not a simple decreasing function of viscosity, but have a lower bound of 0.4142 that dictates the measurement range and sensitivity of the technique. However, in our laboratory, we observe that when the technique is used with highly viscous fluids, reflection coefficients of $<0.4142$ are measured. To interpret our data, we examine the effects of non-Newtonian fluid behavior on reflection coefficient measurement. Results are presented and discussed in this paper.

Non-Newtonian fluids are typically classified into three categories: ${ }^{6}$ (a) time-independent fluids, (b) thixotropic fluids, and (c) viscoelastic fluids. The non-Newtonian behavior of a time-independent fluid is characterized by its nonlinear dependence of shear stress on shear rate. Two phenomena are commonly displayed: shear thinning such as in polymer solutions, and shear thickening such as in solid suspensions. A popular rheological model that describes time-independent fluids is the power-law formula. Thixotropic fluids undergo isotropic change in material properties due to the shearing motion. Little is known of thixotropic fluid phenomena. Viscoelastic fluids usually exhibit a memory for past deformations and hence for past shear; their non-Newtonian behavior can be characterized by relaxation processes, and several mechanical models have been used to describe their phenomena. Commonly used models are based on Maxwell and Voigt models; the Maxwell model has been widely studied.?

\footnotetext{
*Work supported by Office of Environmental Management, U.S. Department of Energy, under Contract W-
} 31-100-ENG-38. 
In this paper, we first derive the expressions of reflection coefficients for the three nonNewtonian fluid models (power-law, Maxwell, and Voigt), and then discuss - on the basis of model calculations - the differences between Newtonian and non-Newtonian fluids. Our measurement technique is then described and data for highly viscous hydrocarbon fluids are presented anc interpreted by the non-Newtonian fluid models.

\section{Theory}

We consider here a practical measurement configuration that utilizes a cylindrical wedge of common industrial materials such as metals or polymers. A shear wave transducer, bonded to the wedge, transmits ultrasonic pulses to the wedge/fluid boundary at a normal incidence angle. Plane shear waves are assumed and the waves are polarized in the direction that the particle motion is parallel to the interface so that mode conversion is avoided. The propagation of the shear waves can be described by

$$
\frac{\partial^{2} \xi}{\partial t^{2}}=\frac{G}{\rho} \frac{\partial^{2} \xi}{\partial y^{2}},
$$

where $\xi$ is the displacement in the $x$ direction, $G$ the shear modulus, and $\rho$ the density of the fluid. In general, $\mathrm{G}$ is a complex quantity and has frequency-dependent real and imaginary components, $G(j \omega)=G(\omega)+j G^{\prime \prime \prime}(\omega)$. For a lossless elastic solid, $G$ is a real quantity so that stress and strain are in phase, but for purely-viscous-or-Newtinian fluids, $G$ is an imaginary quantity given as $G^{\prime \prime}(\omega)=\omega \eta$, where $\eta$ is the fluid shear viscosity. Due to the difference in shear impedance between solid and fluid, shear waves will be partially reflected at the boundary and the reflection coefficient, $R$, is defined as

$$
R=\frac{Z_{w}-Z_{L}}{Z_{w}+Z_{L}},
$$

where $\mathrm{Z}_{\mathrm{w}}$ and $\mathrm{Z}_{\mathrm{L}}$ are the shear impedances of wedge and fluid. respectively. For most wedge materials, $Z_{w}$ is assumed to be a real quantity, $Z_{w}=\left(\rho_{w} C_{44}\right)^{1 / 2}$, where $\rho_{w}$ is the density of wedge and $C_{44}$ is a stiffness constant of the wedge material: For viscoelastic fluids, $Z_{L}$ is a complex quantity $\left(Z_{L}=R_{L}+j X_{L}\right)$, which is defined as

$$
Z_{L}=-\frac{G(\partial \xi / \partial y)}{\partial \xi / \partial t}
$$

Assuming that the displacement, $\xi$, is a sinusoidal function of angular frequency $\omega, Z_{L}$ can also be given as

$$
\mathrm{Z}_{\mathrm{L}}=[\rho \mathrm{G}(\mathrm{j} \omega)]^{1 / 2}
$$

Experimentally, the reflection coefficient is determined by the ratio of absolute magnitudes of reflected signals from the boundary that is in contact with the fluid and from the same boundary when it is exposed to the air. Eq. 2 can be rewritten as 


$$
|R|=\left[\frac{Z_{w}^{2}+R_{L}^{2}+X_{L}^{2}-2 Z_{w} R_{L}}{Z_{w}^{2}+R_{L}^{2}+X_{L}^{2}+2 Z_{w} R_{L}}\right]^{1 / 2}
$$

and

$$
\begin{aligned}
& R_{L}^{2}=\frac{\rho}{2}\left(G+\sqrt{G^{2}+G^{\prime 2}}\right) \\
& X_{L}^{2}=\frac{\rho}{2}\left(-G+\sqrt{G^{2}+G^{\prime 2}}\right)
\end{aligned}
$$

For a purely Newtonian fluid, $G^{\prime}=0$ and $G^{\prime \prime}(\omega)=\omega \eta$, which give

$$
\mathrm{R}_{\mathrm{L}}=\mathrm{X}_{\mathrm{L}}=\sqrt{\frac{\rho \omega \eta}{2}}
$$

In Fig. 1, we show the reflection coefficient as a function of fluid density-viscosity product for different shear-wave frequencies. The dashed lines represent regions where a Newtonian fluid has an impedance greater than the wedge impedance. In reality, such a high-impedance Newtonian fluid does not exist. Thus; the solid-line region of each curve represents the real physical range and therefore the sensitivity that is measurable for that frequency. This Newtonian model indicates that increase of the operating shear-wave frequency will reduce the measurable viscosity range but will increase the measurement sensitivity. It is also noticed that for Newtonian fluids, the reflection coefficient has a lower bound of 0.4142 , which can be easily deduced from Eq. 5 by setting $Z_{w}=2^{1 / 2} R_{L}$.

To examine the effects on reflection coefficient due to non-Newtonian behavior, we choose three commonly used models: Maxwell, Voigt and power-law expressions. The reflection coefficient for each model is derived based on the basis of the definition of shear impedance given by Eq.3. They are summarized below.

\section{A. Maxwell Model}

The Maxwell fluid model relates the shear stress, $\sigma$, and shear rate, $\dot{\gamma}$, by

$$
\sigma+\lambda \dot{\sigma}=\eta \dot{\gamma}
$$

where $\lambda=\eta / G_{\infty}$, a characteristic fluid relaxation time (called Maxwell relaxation time). $G_{\infty}$ is the instantaneous shear elastic modulus as observed at high frequency. If the Maxwell relaxation time of a fluid is comparable to the time over which shear stress is applied, the fluid is considered viscoelastic. If we assume that both shear stress and shear rate vary sinusoidally with angular frequency $\omega$, the complex shear modulus can be given as

$$
\begin{aligned}
& G^{\prime}=\frac{\omega^{2} \lambda \eta}{1+\omega^{2} \lambda^{2}} \\
& G^{\prime \prime}=\frac{\omega \eta}{1+\omega^{2} \lambda^{2}}
\end{aligned}
$$


Substituting Eq.9 into Eqs. 6 and 5, we obtain the reflection coefficient for the Maxwell model. Figure 2 shows reflection coefficients plotted as a function of viscosity for different $G_{\infty}$ values. When $G_{\infty}=10^{15}$, the Maxwell model approaches the Newtonian fluid model. As $G_{\infty}$ decreases, i.e., the Maxwell relaxation time increases if fluid viscosity remains constant, the reflection coefficient will reach a minimum corresponding to total impedance match with the wedge material. This is more clearly illustrated in $\mathrm{Fig}_{\mathrm{g}}$. 3 where we plot the calculated reflection coefficients as a function of $G_{\infty}$ for various fluid viscosities.

\section{B. Voigt Model}

The Voigt model has the following relationship for shear stress and shear rate:

$$
\sigma=G_{\infty} \gamma+\eta \dot{\gamma}
$$

from which we obtain

$$
\begin{aligned}
& G^{\prime}=\frac{G_{\infty}^{3}-G_{\infty} \omega^{2} \eta^{2}}{G_{\infty}^{2}+\omega^{2} \eta^{2}} \\
& G^{\prime \prime}=\frac{\omega \eta\left(\omega^{2} \eta^{2}+3 G_{\infty}^{2}\right)}{G_{\infty}^{2}+\omega^{2} \eta^{2}}
\end{aligned}
$$

Opposite to the Maxwell model, the $G_{\infty}$ of the Voigt model increases as the model deviates from the Newtonian model. Figure 4 shows the reflection coefficients as plotted against viscosity for different $G_{\infty}$ values. The Voigt model approaches the Newtonian model when $G_{\infty}=10^{9}$.

\section{Power Law Model}

The power-law model is widely used to describe time-independent non-Newtonian fluids. The model uses two parameters, $\mathbf{K}$ and $\mathrm{n}$, to relate shear stress and shear rate.

$$
\sigma=K \dot{\gamma}^{n}
$$

By assuming that shear strain $\gamma$ is an oscillatory function, the shear modulus can be given as

$$
\begin{aligned}
& G^{\prime}=-K \omega|\dot{\gamma}|^{n-1} \cos [(n-1) \omega t-n \pi / 2] \\
& G^{\prime \prime}=-K \omega|\dot{\gamma}|^{n-1} \sin [(n-1) \omega t-n \pi / 2]
\end{aligned}
$$

The $(K, n)$ model gives the same expression for Newtonian fluids when $n=1$ and $K=\eta$. For $n<1$ it describes pseudoplastic or shear thinning behavior, while for $n>1$ it is used to describe dilatant or shear thickening behavior. Figure 5 shows the reflection coefficient functions for the power-law model. Clearly, the viscosity range covered by the reflection coefficient measurement increases as $\mathrm{n}$ increases.

\section{Experiment}


In principle, to determine the reflection coefficient, one must measure both amplitude and phase of the reflected signals. In practice, the phase change is very small and requires high-frequency operation for accurate determination. In our experiments, only the amplitude information is used to determine the reflection coefficient which is given by the amplitude ratio of the reflected signals from the wedge/ uid and reference interfaces. We choose the air/wedge boundary as the reference interface because a total reflection from this interface can be assumed.

A two-step wedge, as shown in Fig. 6, was designed to produce the two reflections from the same incident signal, and the ratio of the two signals gives a measure of the reflection coefficient. Measurement sensitivity and accuracy of this technique are very dependent on wedge material. A high-impedance material such as stainless steel gives poor sensitivity and large measurement error. For viscosity measurement, the theoretical error can be estimated from the following expression:

$$
\frac{\Delta \eta}{\eta}=\frac{4 R_{S}}{1-R_{S}^{2}} \frac{\Delta R_{S}}{R_{S}}
$$

where $R_{s}$ represents the shear-wave reflection coefficient. Equation 14 indicates that measurement accuracy depends on the reflection coefficient itself. Hence, for wedges of high shear impedance, which causes a large impedance mismatch with most fluids of interest, the viscosity measurement not only lacks sensitivity but also introduces a large measurement error. Selection of a proper wedge material is the critical step in the reflectioncoefficient measurement; Table 1 lists the characteristics of some candidate materials. The pulse-echo method was used to measure both the longitudinal and shear velocities of the wedges. Figure 7 shows calculated reflection coefficients for several wedge materials. It is clearly shown that polymeric materials are suggested for better sensitivity while metallic materials are suitable for a wider measurement range of viscosity. A Lucite wedge was chosen for this experiment and measurements were conducted under static conditions and with shear-wave frequency of $5 \mathrm{MHz}$.

Reflection coefficient measurements were made for hydrocarbon-based viscosity standards provided by Cannon Instrument $\mathrm{Co}$. The densities of the standards range from 0.85 to 0.91 , while viscosity covers a range from 1381 to $5.7 \times 10^{6} \mathrm{cP}$. The standards are labeled as Newtonian fluids. However, we found that some of the highly viscous standards give reflection coefficients below the lower limit of the Newtonian-fluid value $(0.4142)$, and their reflection coefficients can be better fitted with non-Newtonian fluid models. The measured data show three regions, as shown in Fig. 8: (a) the low-viscosity fluids $\left(<10^{4}\right.$ cP) can be fitted into the Newtonian model, (b) the highly viscous fluids $\left(>2 \times 10^{5} \mathrm{cP}\right)$ can be described by the Maxwell model with $G_{\infty}=3.8 \times 10^{11}$, and (c) the intermediate region fits the power-law model with $n=1.09$.

\section{Conclusions}

Measurement of reflection coefficient at a solid/fluid interface has proven to be reliable and simple in determining fluid shear viscosity under steady flow conditions. In this paper, we examine the effects on reflection coefficient due to non-Newtonain fluid behavior. Identification of such effects can be useful in characterizing non-Newtonian fluids. We derived the expressions of reflection coefficient for both Newtonian and non-Newtonian fluid models. Three commonly used non-Newtonian models are included: Maxwell, Voigt, and power-law. Based on our model calculations, we found that (a) Newtonian fluids show a lower bound of reflection coefficient (0.4142), (b) the Maxwell model predicts lower reflection coefficients if a proper relaxation time is chosen, (c) the Voigt model 
shows a higher measurement sensitivity in low-viscosity regions, and (d) the power-law model shows a smooth transition from pseudoplastic to dilatant fluids. Reflection coefficients were measured with a Lucite wedge for the Cannon viscosity standards. The results show that for those standards of high viscosity $\left(>2 \times 10^{5} \mathrm{cP}\right)$, the Maxwell model gives a satisfactory interpretation, while the power-law model can describe the data for the standards of viscosity in the range of $10^{4}$ to $10^{5} \mathrm{cP}$. Thus, we have shown that it is possible to characterize non-Newtonian fluid behavior by measuring the reflection coefficient at a solid/fluid interface.

\section{References}

1. R. S. Moore and H. J. McSkimin, "Dynamic Shear Properties of Solvents and Polystyrene Solutions from 20 to $300 \mathrm{MHz}$," Physical Acoustics, vol. VI, pp. 167-243, 1970.

2. G. Harrison and A. J. Barlow, "Dynamic Viscosity Measurement," Methods of Experimental Physics, vol. 19, pp. 138-180, 1981.

3. M. J. Lea, and P. Fozooni, "The Transverse Acoustic Impedance of an Inhomogeneous Viscous Fluid," Ultrasonics International 85, Butterworths, pp. 133-136, 1985.

4. L. C. Lynnworth, Ultrasonic Measurements for Process Control, Academic Press, Inc., p. 50, 1989.

5. S. H. Sheen, H. T. Chien, and A. C. Raptis, "An In-line Ultrasonic Viscometer," Rev. of Progress in Quantitative Nondestructive Evaluation, vol. 14A, pp. 1151-1158, 1995.

6. J. Harris, Rheology and Non-Newtonian Flow, Longman Group Limited, 1977, ch. 1.

7. G. B. Thurston, "Shear Rate Dependence of the Viscoelasticity of Polymer Solutions," J. of Non-Newtonian Fluid Mechanics, vol. 9, pp. 57-68, 1981. 


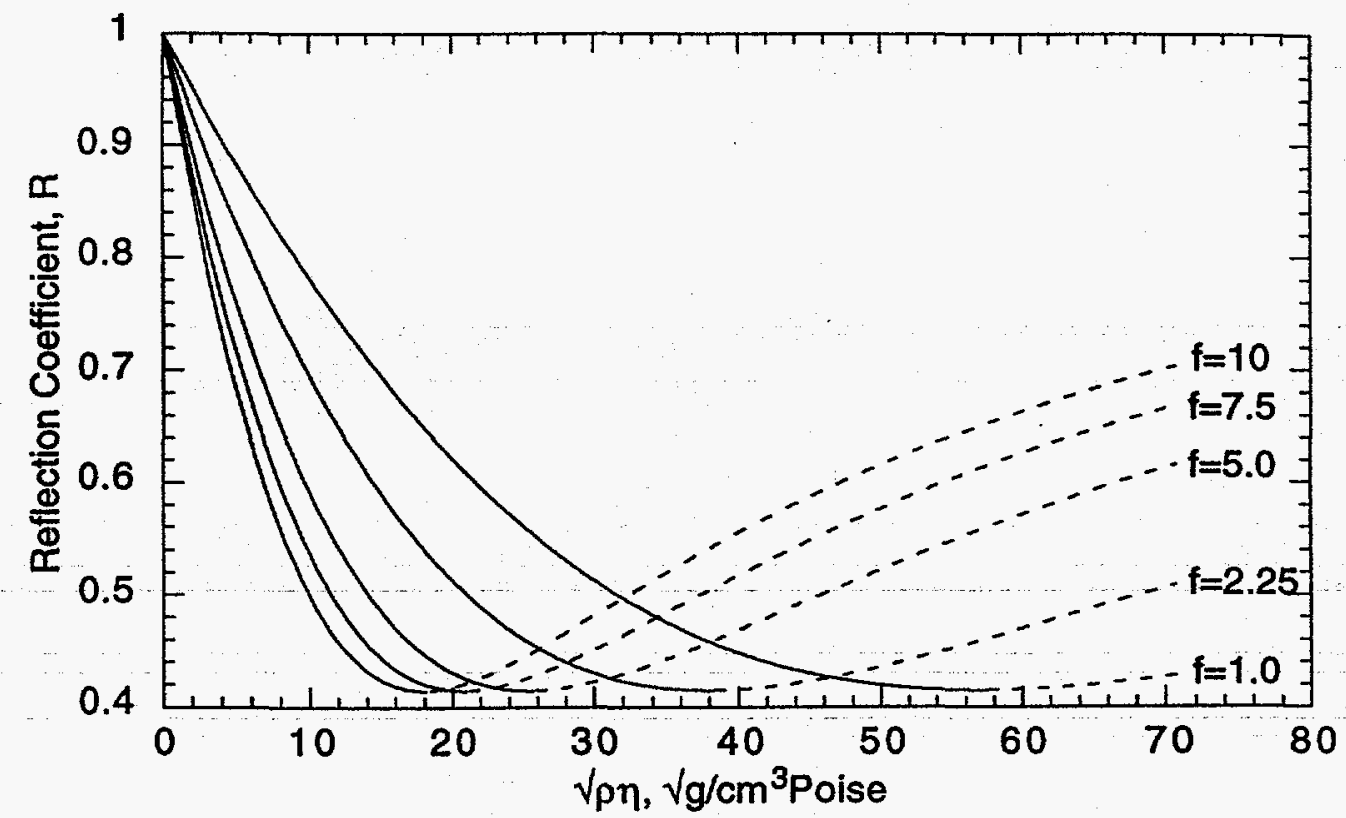

Fig. 1: Reflection coefficient as a function of fluid density-viscosity product for different operating shear wave frequencies. 


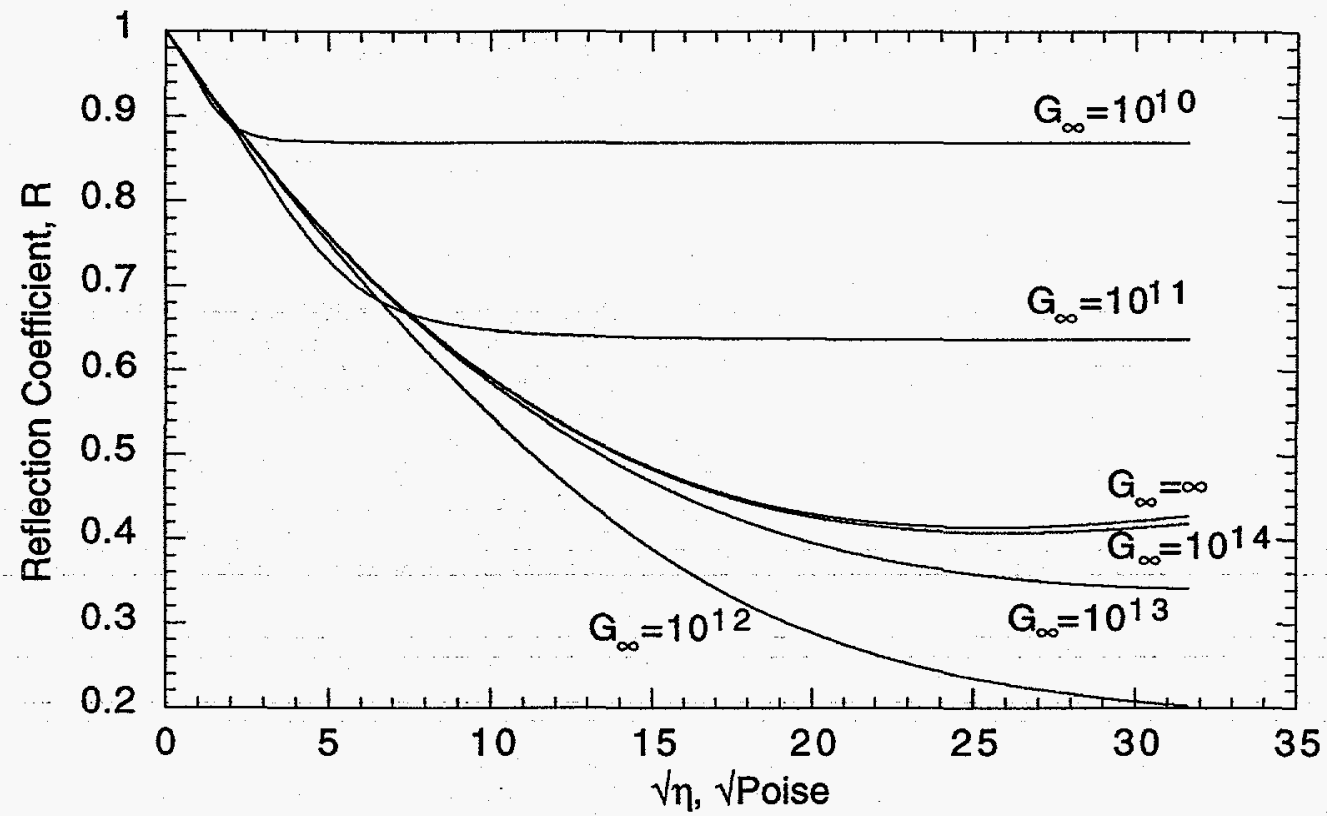

Fig. 2: Reflection coefficient as a function of fluid viscosity for Maxwell models of different shear moduli, $G_{\infty}$. 


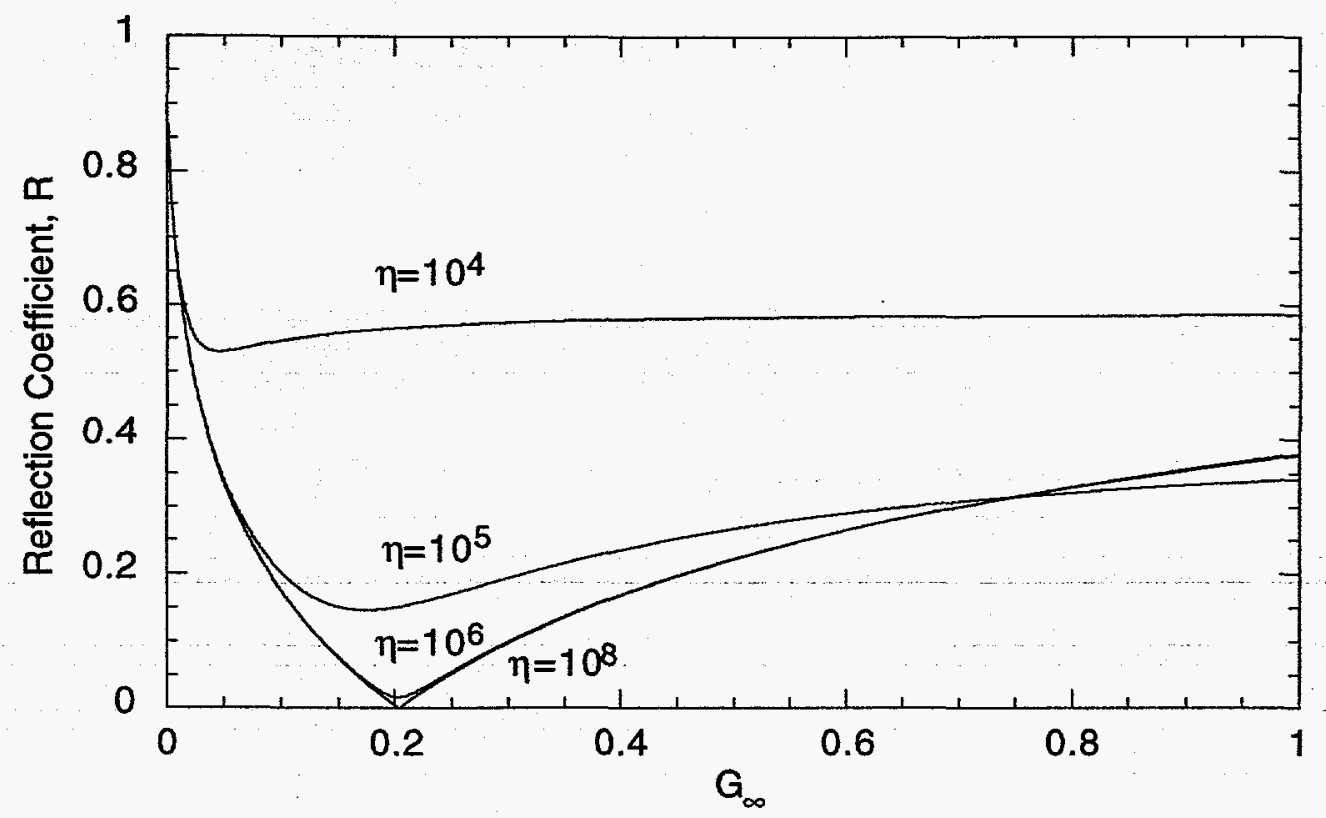

Fig. 3: Reflection coefficient as a function of shear modulus, $G_{\infty}$, for Maxwell models of different shear viscosities, in Poise. 


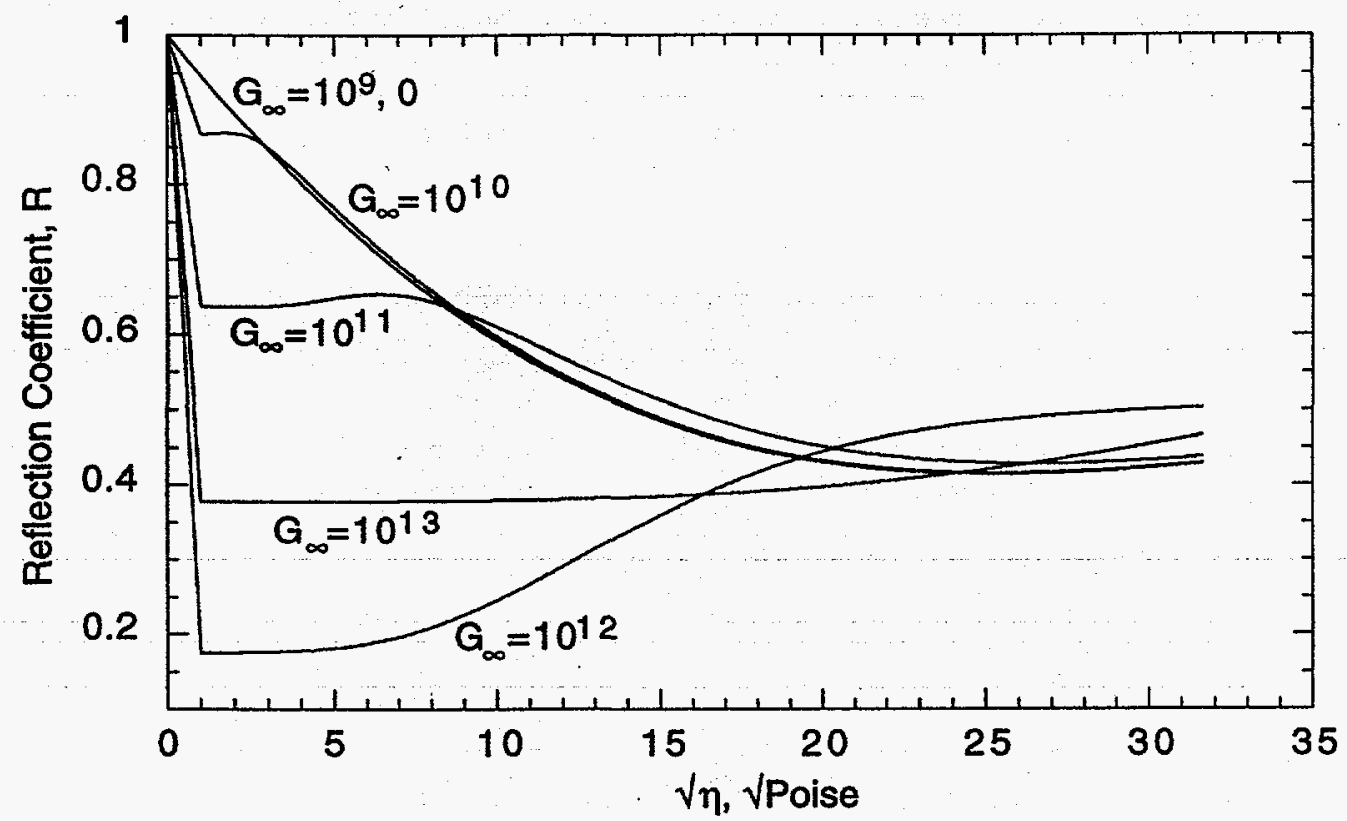

Fig. 4: Reflection coefficient as a function of fluid viscosity for Voigt models of different shear elastic modulus, $G_{\infty}$. 


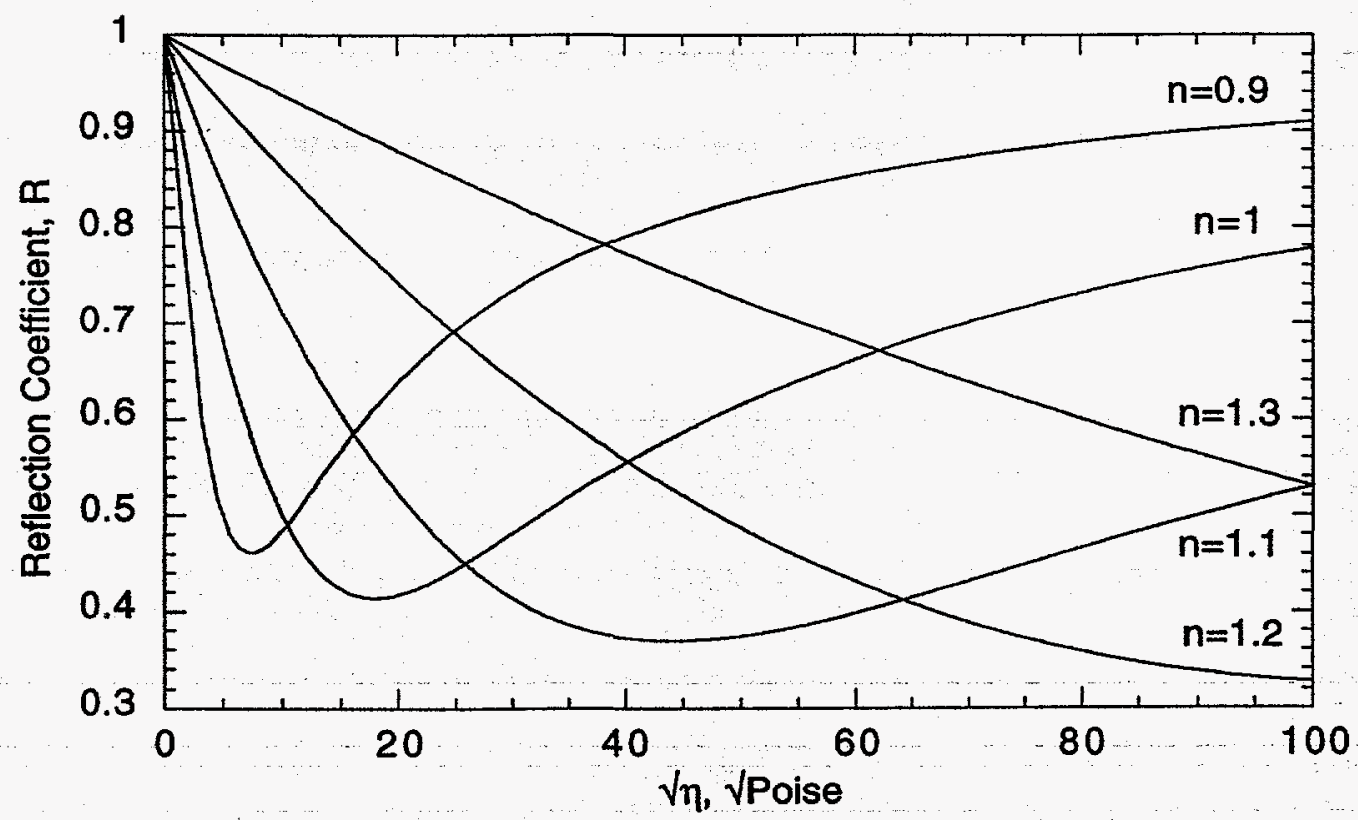

Fig. 5: Reflection coefficient as a function of sqrt root of fluid viscosity for power-law models of different $n$ values. 
Table 1: Characteristics of different wedge materials.

\begin{tabular}{||l|c|c|c|c|c|c|}
\hline \multicolumn{1}{|c|}{ Material } & $\begin{array}{c}\text { Density } \\
\rho\left(\mathrm{g} / \mathrm{cm}^{3}\right)\end{array}$ & $\begin{array}{c}\text { Long. Velocity } \\
\mathrm{V}_{\mathrm{L}}(\mathrm{cm} / \mu \mathrm{s})\end{array}$ & $\begin{array}{c}\text { Long. Impedance } \\
\rho \mathrm{V}_{\mathrm{L}}\end{array}$ & $\begin{array}{c}\text { Shear Velocity } \\
\mathrm{V}_{\mathrm{SH}}(\mathrm{cm} / \mu \mathrm{s})\end{array}$ & $\begin{array}{c}\text { Shear Impedance } \\
\rho \mathrm{V}_{\mathrm{SH}}\end{array}$ & $\begin{array}{c}\text { Working Temp. } \\
\mathrm{T}_{\mathrm{w}}\left({ }^{\mathrm{o}} \mathrm{F}\right)\end{array}$ \\
\hline ABS $^{\top}$ & 1.5279 & 0.2330 & 0.3560 & - & 185 \\
\hline Acrylic (Cast) & 1.1800 & 0.2731 & 0.3222 & 0.1369 & 0.1615 & 200 \\
\hline Acrylic (Extruded) & 1.1800 & 0.2525 & 0.2979 & 0.1369 & 0.1615 & 200 \\
\hline Delrin & 1.0341 & 0.2137 & 0.2210 & 0.0931 & 0.0963 & 180 \\
\hline Lucite & 1.2800 & 0.2335 & 0.2989 & 0.1119 & 0.1432 & 200 \\
\hline Plexiglass & 1.1897 & 0.2701 & 0.3214 & 0.1621 & 0.1928 & 200 \\
\hline Polyetherimide & 1.2700 & 0.2403 & 0.3052 & 0.1041 & 0.1323 & 338 \\
\hline Polystyrene & 1.0279 & 0.2042 & 0.2099 & - & - & 170 \\
\hline WTD $^{*}$ & 1.2624 & 0.2352 & 0.2969 & 0.1016 & 0.1283 & 350 \\
\hline HTD $^{*}$ & 1.4038 & 0.2591 & 0.3637 & 0.1127 & 0.1582 & 500 \\
\hline VHTD $^{*}$ & 1.4315 & 0.2309 & 0.3305 & 0.0985 & 0.1409 & 900 \\
\hline
\end{tabular}

${ }^{+}$ABS: Acrylonitrile-butadiene-styrene

* Delay lines are supplied by Panametric, Inc. for high-temperature applications. WTD: Moderate Temperature Delay line; HTD: High Temperature Delay line; VHTD: Very High Temperature Delay line 


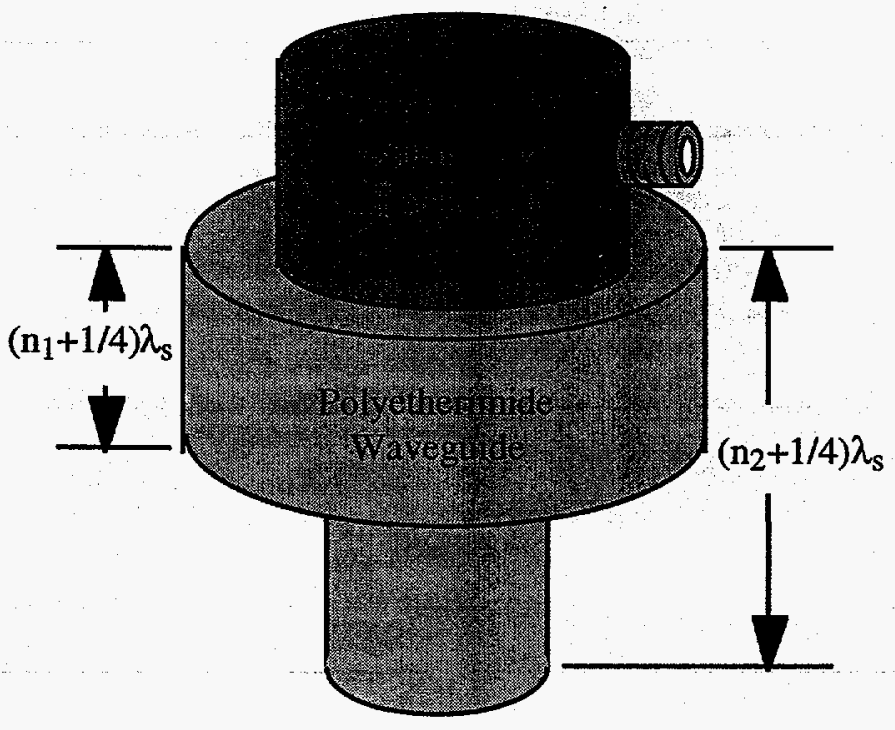

Fig. 6: Two-step wedge design, Ls: wavelength of shear wave in wedge. 


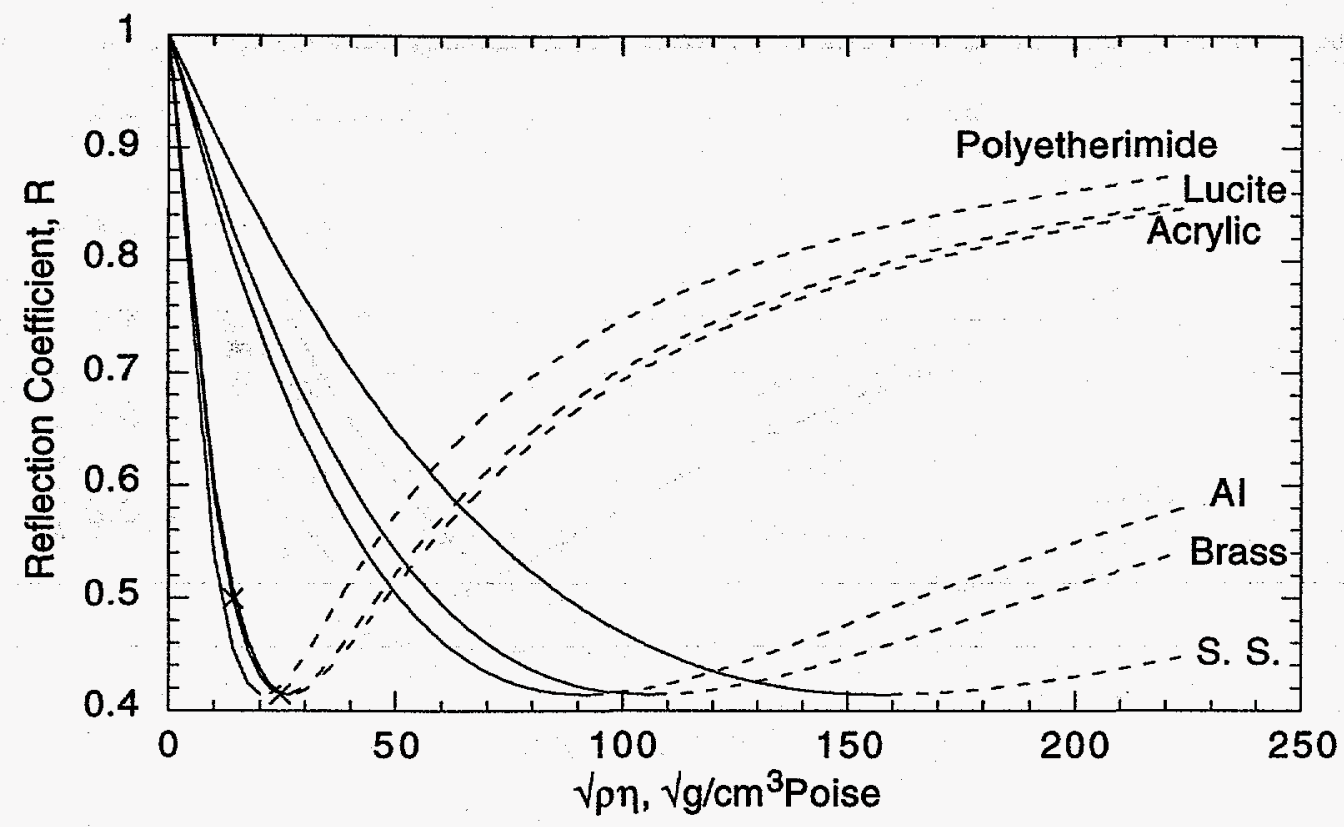

Fig. 7: Reflection coefficient Vs. square root of fluid density-viscosity product for different wedge materials. 


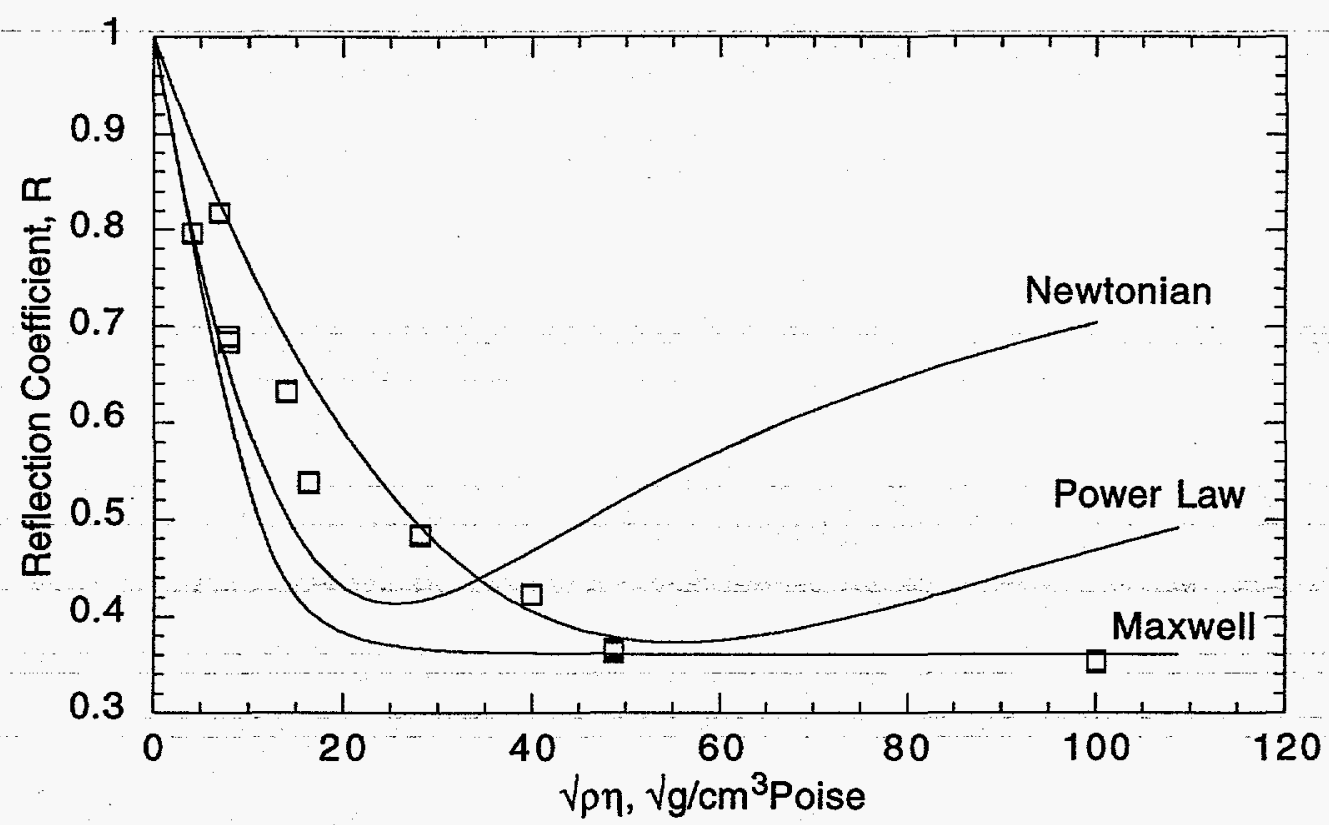

Fig. 8: Measured reflection coefficient for Cannon viscosity standards and calculated values using Maxwell model with $\mathrm{G}_{\infty}=3.8 \times 10^{11}$ and power-law model with $\mathrm{n}=$ 1.09 . 